\title{
Fluorescent In Situ Hybridization Analysis of Cecal Microflora in Rats Simultaneously Administrated Lactobacillus rhamnosus KY-3 and Cellobiose
}

\author{
Miki UmeKi ${ }^{1}$, Kazutoshi Out ${ }^{1}$, Masatsugu Mori², Satoshi Mochizuki ${ }^{3}$ and Kenji SAKAI ${ }^{1 *}$ \\ ${ }^{1}$ Department of Applied Chemistry, Faculty of Engineering, Oita University, 700 Dannoharu, Oita, 870-1192, Japan \\ ${ }^{2}$ Eco Up Company, Ltd., 3905-1, Katata, Saiki-shi, Oita, 876-1512, Japan \\ ${ }^{3}$ Faculty of Education and Welfare Science, Oita University, 700 Dannoharu, Oita, 870-1192, Japan
}

Received July 14, 2004; Accepted February 25, 2005

\begin{abstract}
The cecal microflora of rats coadministered Lactobacillus rhamnosus KY-3 (L. rhamnosus KY-3) and cellobiose was analyzed by fluorescence in situ hybridization. When compared with L. rhamnosus KY-3 administration alone, simultaneous administration of $L$. rhamnosus KY-3 and cellobiose led to an increase in the number of lactic acid bacteria (LAB), particularly L. rhamnosus, and a significant decrease in the number of Gammaproteobacteria in the cecum. These results indicate that administration of L. rhamnosus KY-3 cells passed through the upper digestive tract to the cecum where it is likely that they proliferated through the assimilation of cellobiose.
\end{abstract}

Keywords: Lactobacillus rhamnosus, cellobiose, microflora, FISH, cecum, synbiotics

\section{Introduction}

We previously proposed a recycling process for municipal food waste based on polylactic acid production (Sakai et al., 2004). In an attempt to achieve zero emissions for the entire recycling process, we have continued conducting research into reducing and reusing the byproducts of this process. One of the byproducts of the lactic acid produced with our proposed process is the use of Lactobacillus rhamnosus KY-3 (L. rhamnosus KY-3) as a feed additive.

The combination of pre- and probiotics has been termed "synbiotics" (Roberfroid, 1998) due to their potential for synergy. We investigated L. rhamnosus KY-3 for use in synbiotics. Cellobiose was chosen to examine the efficacy of coadministration of probiotic microflora with an indigestible oligosaccharide for use as food. In a study using rats, when L. rhamnosus KY-3 (probiotic) was coadministered with cellobiose (prebiotic), concentrations of serum total lipids, triacylglycerol, cholesterol, and phospholipids decreased. However, the decrease in these serum lipids was not observed when L. rhamnosus KY-3 was administered alone (Umeki et al., 2004). In order to better understand the mechanisms of such decreases in serum lipids in synbiotic-administered rats, it may be prove useful to analyze changes in the intestinal microflora.

Fluorescence in situ hybridization (FISH), which targets specific rRNA sequences, facilitates direct microscopic observation of microorganisms and the gathering of phylogenetic information. We have been using FISH to identify changes in complex microbial flora, such as those seen during open lactic acid fermentation of minced

E-mail: sakai@cc.oita-u.ac.jp kitchen refuse (Sakai et al., 2004). Given that FISH is also effective for determining intestinal flora in animals, 16S rDNA oligonucleotide probes specific to the lactic acid bacteria (LAB), L. plantarum, Lactococcus lactis, and L. rhamnosus have been developed (Sakai et al., submitted). Using these probes, we analyzed the cecal microflora in rats receiving $L$. rhamnosus KY-3 and then confirmed the optimal hybridization conditions for detecting LAB and L. rhamnosus.

In the present study, we employed the above-mentioned technique in an effort to determine the effects of synbiotic administration (L. rhamnosus KY-3 and cellobiose) on the intestinal flora of rats.

\section{Materials and Methods}

L. rhamnosus KY-3 was grown to the stationary phase in MRS medium (Atras, 1993) at $37^{\circ} \mathrm{C}$ for $24 \mathrm{~h}$ under anaerobic conditions. Cells collected by centrifugation $\left(10,000 \times \mathrm{g}\right.$ for $20 \mathrm{~min}$ at $\left.4^{\circ} \mathrm{C}\right)$ were pelleted and stored at $-80^{\circ} \mathrm{C}$ (without stabilizer) until diet preparation. Cellobiose was obtained from Matsutani Chemical Industry Co., Ltd. (Itami, Japan).

We purchased 3-week-old male Wistar rats from Japan SLC Inc. (Hamamatsu, Japan). Rats were housed in individual cages with stainless steel screen bottoms in a room maintained at $23 \pm 1^{\circ} \mathrm{C}$ and $55 \pm 5 \%$ relative humidity, with a 12-h light (0800-2000 h) and dark cycle. This study conformed to the guidelines for the care and use of laboratory animals (Notification No. 6 of the Prime Minister's Office, March 27, 1980).

Rats were acclimatized to a stock diet (5L37; Japan SLC Inc., Hamamatsu, Japan) for 3 days. After acclimatization, rats were divided into 4 groups consisting of 6 rats according to body weight. Rats on the control diet were 
fed on a diet containing $20 \%$ casein (control diet: casein $200 \mathrm{~g} / \mathrm{kg}$; DL-methionine $3 \mathrm{~g} / \mathrm{kg}$; sucrose $682 \mathrm{~g} / \mathrm{kg}$; soybean oil $70 \mathrm{~g} / \mathrm{kg}$; AIN93G mineral mix $35 \mathrm{~g} / \mathrm{kg}$; AIN93 $\mathrm{G}$ vitamin $\operatorname{mix} 10 \mathrm{~g} / \mathrm{kg}$ ), supplemented with either $L$. rhamnosus KY-3 (1.7\% dry weight; $11 \%$ wet weight; $2 \times$ $10^{13}$ cells $\left./ \mathrm{kg}\right), 10 \%$ cellobiose $(\mathrm{CEB})$ or $1.7 \%$ L. rhamnosus KY -3 and $10 \%$ cellobiose (KY-3+CEB). Food and water were provided ad libitum for 13 days.

At the end of the 13-day period, each rat was euthanized and the cecum was removed. Cecal contents were analyzed by FISH according to the method of Amann et al. (Amann, 1995) under a fluorescence microscope in order to determine the number of all bacteria, most LAB (including lactobacilli and lactococci), L. rhamnosus and Gammaproteobacteria using the 16S rDNA probes, EUB338 (Amann et al., 1990), LAC722 (Sakai et al., 2004), Lrham454 (Sakai et al., submitted) and GAM42a (Manz et al., 1992), respectively. The number of bacterial cells was deter- mined according to the method of Sakai et al. (Sakai et al., 2004).

Data are expressed as means \pm standard error. Data were statistically analyzed by one-way ANOVA. When differences between treatments were significant $(\mathrm{p}<0.05)$, Duncan's new multiple range test was performed (Duncan, 1957).

\section{Results and Discussion}

Figure 1 shows cell numbers of cecal bacteria, as assessed by FISH. When compared with the control group, the number of most LAB in the KY-3 group and the CEB group was 18- and 4-fold higher, respectively. The numbers of all bacteria and most LAB in the KY-3+CEB group were significantly higher than those in the other groups; the number of most LAB in the KY-3+CEB group was 40 -fold higher than that in the control group. When compared with the control group, the number of $L$.
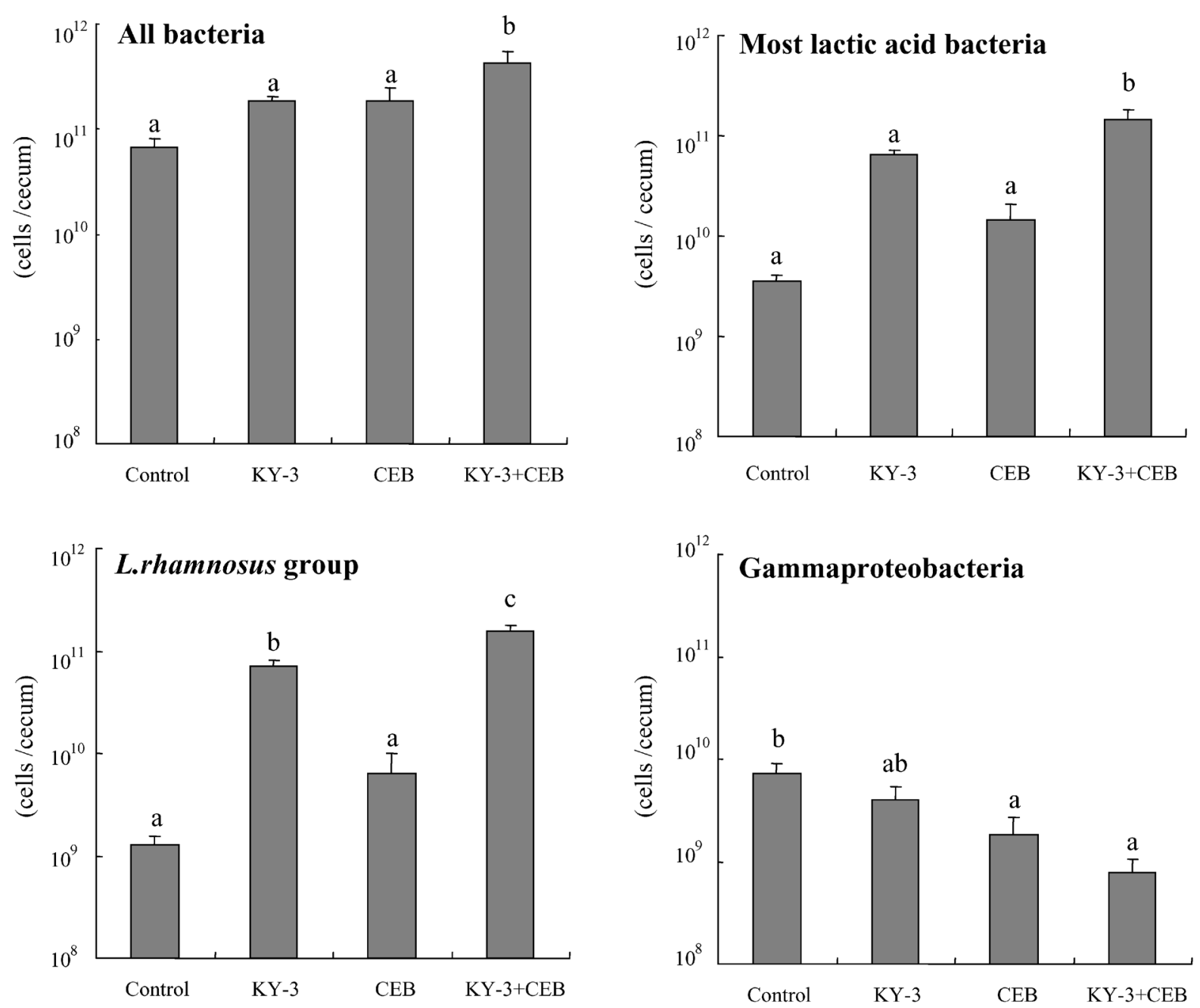

Fig. 1. Populations of bacteria isolated from cecal contents of rats administrated L. rhamnosus KY-3 and cellobiose. Rats were fed on $20 \%$ casein diets (Control) or experimental diets containing $1.7 \%$ dry weight (11\% wet weight, $2 \times 10^{13}$ cells $/ \mathrm{kg}$ diet) of L. rhamnosus KY-3 (KY-3), 10\% cellobiose (CEB) or 1.7\% L. rhamnosus KY-3 and 10\% cellobiose (KY-3+CEB). All bacterial cells were enumerated by FISH with the EUB338 probe, while most lactic acid (including lactobacilli and lactococci) bacterial cells, L. rhamnosus cells and Gammaproteobacterial cells were enumerated by FISH using the LAC722 (L), Lrham454 and GAM42a probes, respectively. Values are expressed as means \pm standard error $(n=6)$, and values not sharing a common letter are significantly different $(\mathrm{p}<0.05)$ as analyzed by Duncan's multiple-range test. 
rhamnosus in the KY-3 group was significantly higher, with levels in the KY-3 + CEB group being the highest (120 times). The relative number of cells stained with Lrham 454 against EUB338 was markedly higher in the KY-3 group (39.0\%) and the KY-3+CEB group (47.3\%) compared to the control group $(2.1 \%)$. No corresponding elevation was observed in the CEB group (3.1\%). Interestingly, the relative number of $L$. rhamnosus cells in the KY-3+CEB group was slightly elevated as compared to the KY-3 group, implying that the increased numbers of LAB in these two groups could be attributed to L. rhamnosus administration, and thus that $L$. rhamnosus $\mathrm{KY}-3$ reaches the intestine without being entirely digested. Furthermore, the number of Lrham454-positive bacterial cells was highest in the KY-3+CEB group, suggesting that $L$. rhamnosus may be able to proliferate by assimilating cellobiose.

The results of the present study showed that the number of Gammaproteobacteria in the CEB and KY-3+ CEB groups was significantly lower than the control group. The number of Gammaproteobacteria in the KY$3+$ CEB group was the lowest among the four groups, and was only one tenth that of the control group. The relative number of cells stained with GAM45a against EUB 338 in the $\mathrm{KY}-3+\mathrm{CEB}$ group $(0.3 \%)$ was significantly lower than the control group $(14.4 \% ; 1 / 50)$ indicating that a decrease in Gammaproteobacteria was involved in the reduced levels of serum lipids. In 1987, Silva et al. reported that L. rhamnosus GG suppressed the growth of numerous microorganisms, including Escherichia coli, by producing a specific antibiotic. Consequently, future research should focus on isolation of such an antibiotic from L. rhamnosus KY-3.

The results of the present study corroborate the hypothesis that synbiotic administration is effective in increasing the numbers of most LAB and decreasing the number of the Gammaproteobacteria to which the Gramnegative coliform bacteria belong. Given that this type of improvement in intestinal microflora may be involved in reducing serum lipid levels, L. rhamnosus KY-3 may be more useful when administered in the form of a preprobiotic (synbiotic) with an indigestible oligosaccharide. Use of L. rhamnosus KY-3 in pet and cattle feeds appears to be useful for preventing lifestyle-related diseases in pets and neonatal diarrhea caused by E. coli in livestock. Further studies, as well as analyses of microflora by FISH, are thus necessary for investigating the effects of synbiotic LAB administration in pets and livestock.

Acknowledgments The authors are grateful to Matsutani Chemical Industry Co., Ltd. for generously providing cellobiose. This study was supported by Special Coordination Funds and by a Grant-in-Aid for Scientific Research (No. 14360202) from the Ministry of Education, Culture, Sports, Science and Technology of Japan.

\section{References}

Amann, R., Krumholz, L. and Stahl, D.A. (1990). Fluorescentoligonucleotide probing of whole cells for determinative, phylogenetic and environmental studies in microbiology. J. Bacteriol., 172, 762-770.

Amann, R. (1995). In situ identification of microorganisms by whole cell hybridization with rRNA-targeted nucleic acid probes. Mol. Microb. Ecol. Manual, 3.3.61-65., Kluwer Academic Publisher.

Atlas, R.M. (1993). "Handbook of microbiological medi” ed. by L.C. Parks. CRC Press Inc., Florida.

Duncan, D.B. (1957). Multiple range tests for correlated and heteroscedastic means. Biometrics, 13, 164-167.

Manz, W., Amann, R., Ludwig, W., Wagner, M. and Schleifer, K.H. (1992). Phylogenetic oligonucleotide probes for the major subclass of proteobacteria: problems and solutions, 15, 593-600.

Roberfroid, M.(1998). Prebiotics and synbiotics: Concepts and nutritional properties. Br. J. Nutr., 80 (suppl. 2), S197-202

Sakai, K., Taniguchi, M., Miura, S., Ohara, H., Matsumoto, T. and Shirai, Y. (2004). Making plastics from garbage: A novel process for poly-L-lactate production from municipal food waste. $J$. Indust. Ecol., 7, 63-74.

Sakai, K., Mori, M., Fujii, A., Iwami, Y., Chukeatirote, E. and Shirai, Y. (2004). Fluorescent in situ hybridization analysis of open lactic acid fermentation of kitchen refuse using rRNAtargeted oligonucleotide probes. J. Biosci. Bioeng., 98, 48-56.

Sakai, K., Oue, K., Umeki, M., Mori, M. and Mochizuki, S. Speciesspecific FISH analysis of cecal microflora in rats administered with lactic acid bacteria. World J. Microbiol. Biotechnol., (accepted).

Silva, M., Jacobus, N.V., Deneke, C. and Gorbach, S.L. (1987). Antimicrobial substance from a human Lactobacillus strain. Antimicrob. Agents. Chemother., 31, 1231-1233.

Umeki, M., Oue, K., Mochizuki, S., Shirai, Y. and Sakai, K. (2004). Effect of Lactobacillus rhamnosus KY-3 and cellobiose as synbiotics on lipid metabolism in rats. J. Nutr. Sci. Vitaminol., 50, 330-334. 